\title{
1 Signatures of optimal codon usage predict metabolic ecology in budding yeasts
}

2 Abigail Leavitt LaBella

3 Department of Biological Sciences, Vanderbilt University, Nashville, TN 37235, USA

4 ORCID: 0000-0003-0068-6703

$5 \quad$ Abigail.1.1abella@vanderbilt.edu

7 Dana A. Opulente

8 Laboratory of Genetics, DOE Great Lakes Bioenergy Research Center, Wisconsin Energy

9 Institute, Center for Genomic Science Innovation, J.F. Crow Institute for the Study of Evolution,

10 University of Wisconsin-Madison, Madison, WI 53726, USA

12 Jacob Steenwyk

13 Department of Biological Sciences, Vanderbilt University, Nashville, TN 37235, USA

14 ORCID: 0000-0002-8436-595X

15 Jacob.steenwyk@vanderbilt.edu

17 Chris Todd Hittinger

18 Laboratory of Genetics, DOE Great Lakes Bioenergy Research Center, Wisconsin Energy

19 Institute, Center for Genomic Science Innovation, J.F. Crow Institute for the Study of Evolution,

20 University of Wisconsin-Madison, Madison, WI 53726, USA

21 ORCID: 0000-0001-5088-7461

22 cthittinger@wisc.edu

24 Antonis Rokas

25 Department of Biological Sciences, Vanderbilt University, Nashville, TN 37235, USA

26 ORCID: 0000-0002-7248-6551

27 antonis.rokas@vanderbilt.edu

28 Running head: Codon usage as a lens into the metabolic ecology of budding yeasts

29 Keywords: Codon usage bias; Budding yeasts; Secondary metabolism; Reverse Ecology 


\section{ABSTRACT}

31 Reverse ecology is the inference of ecological information from patterns of genomic variation.

32 One rich, heretofore underutilized, source of ecologically-relevant genomic information is codon

33 optimality or adaptation. Bias toward codons that match the tRNA pool is robustly associated

34 with high gene expression in diverse organisms, suggesting that codon optimization could be

35 used in a reverse ecology framework to identify highly expressed, ecologically relevant genes.

36 To test this hypothesis, we examined the relationship between optimal codon usage in the classic

37 galactose metabolism $(G A L)$ pathway and known ecological niches for 329 species of budding

38 yeasts, a diverse subphylum of fungi. We find that optimal codon usage in the GAL pathway is

39 positively correlated with quantitative growth on galactose, suggesting that $G A L$ codon

40 optimization reflects increased capacity to grow on galactose. Optimal codon usage in the $G A L$

41 pathway is also positively correlated with human-associated ecological niches in yeasts of the

42 CUG-Ser1 clade and with dairy-associated ecological niches in the family Saccharomycetaceae.

43 For example, optimal codon usage of $G A L$ genes is greater than $85 \%$ of all genes in the major

44 human pathogen Candida albicans (CUG-Ser1 clade) and greater than $75 \%$ of genes in the dairy

45 yeast Kluyveromyces lactis (family Saccharomycetaceae). We further find a correlation between

46 optimization in the thiamine biosynthesis and GAL pathways. As a result, optimal codon usage in

47 thiamine biosynthesis genes is also associated with dairy ecological niches in

48 Saccharomycetaceae, which may reflect competition with co-occurring microbes for

49 extracellular thiamine. This work highlights the potential of codon optimization as a tool for

50 gaining insights into the metabolic ecology of microbial eukaryotes. Doing so may be especially

51 illuminating for studying fungal dark matter - species that have yet to be cultured in the lab or

52 have only been identified by genomic material. 


\section{INTRODUCTION}

54 The immense diversity of life is due, in part, to adaptation to the wide variety of environmental

55 niches available. By acting on the interface between genotype, phenotype, and environment,

56 natural selection has given rise to numerous ecological adaptations [1-3]. The precise

57 relationship between genotype, phenotype, and environment, however, is often elusive. For

58 example, a connection was only recently made between environmental distribution of seeds of

59 different sizes, phenotypic variation in the beaks of Darwin's finches, and changes in the

60 expression of the protein BMP4 [4-6].

61 Genomic sequencing has accelerated the rate at which the underlying genomic mechanisms of

62 well-established ecologically adapted phenotypes are elucidated [7,8]. While powerful, this type

63 of ecological genomics requires extensive knowledge of the ecological niche in which species

64 live. For many microbial species, however, detailed ecological information is unavailable due to

65 both the scale of the ecosystems they live in and the dearth of information reported during

66 collection [9]. One potentially powerful way to address this gap in knowledge is to use the

67 extensive genomic resources available in microbes to conduct reverse ecology - directly

68 inferring ecology from genotype [10,11].

69 Reverse ecology has successfully linked environmental phenotype with genotype using multiple

70 types of genomic features [11-13]. Optimal growth temperature was successfully inferred from

71 genomic content, including tRNA, ribosome, and gene features, in 549 Bacteria and 170 Archaea

72 [14]. In the red bread mold Neurospora crassa, analysis of highly divergent genomic regions in

7348 isolates uncovered "genomic islands" associated with adaptation in two different ecosystems

74 [15]. Across the entire tree of life, metabolic capability (assessed using Kyoto Encyclopedia of 
75 Genes and Genomes (KEGG) gene annotations) was used to examine the evolution of

76 exogenously required metabolites likely found in the environment [16]. Metabolic network

77 analysis has emerged as a common genomic feature for reverse ecology analysis [17,18]. There

78 are, however, other promising genomic features that can be used in reverse ecology.

79 One underutilized genomic feature with great potential for reverse ecology studies is codon

80 usage, which has long been associated with gene expression [19-21]. Changes in gene

81 expression have been shown to play an important role in ecological adaptation [22-24]. For

82 example, in wild isolates of budding yeast Saccharomyces cerevisiae, changes in the expression

83 of multiple genes were associated with phenotypic differences in copper resistance and

84 pigmentation that may be associated with high copper environments [25]. Over evolutionary

85 time, increased levels of gene expression result in a selective pressure for accurate and efficient

86 translation [26-30] and increased mRNA stability [31,32]. Codons that match the tRNA pool-

87 called optimal codons - have a substantial impact on both translation [27,29,30] and mRNA

88 stability [31]. Therefore, optimal codon usage is correlated with high gene expression in multiple

89 lineages, especially in microbes [19,33-38]. Therefore, we hypothesize that ecological

90 adaptations that are, at least partly, due to high expression levels of specific genes or pathways

91 will be reflected in their codon usage values.

92 Previous work in diverse microbes supports the hypothesis that codon optimization can be used

93 to identify associations between codon usage (either globally or in specific genes) and ecology

94 [12,39-43]. For example, an analysis of metagenomes collected from mine biofilms shows an

95 enrichment of optimal codons in bacterial and archaeal genes associated with inorganic ion

96 transport[39]. In fungi, codon optimization in host-induced and secreted proteins is associated

97 with generalist fungal parasites [41]. Although these studies were highly successful in linking 
98 particular ecological niches with highly enriched groups of genes, we still lack examples where

99 reverse ecology has linked particular ecologies to specific pathways.

100 The galactose (GAL) pathway (also known as the Leloir pathway) in the budding yeast

101 subphylum Saccharomycotina is an iconic pathway that metabolizes galactose into glucose,

102 which can then be used in core metabolism or as an intermediate $[44,45]$. The genes encoding the

103 three enzymes of the GAL pathway_GAL1 (encoding a galactokinase), GAL10 (encoding a

104 UDP-glucose-4-epimerase), and GAL7 (encoding a galactose-1-phosphate uridyl transferase)—

105 are frequently clustered in yeast genomes and are induced in response to the presence of

106 galactose [46-48]. There has been extensive research into the biochemistry [44], regulation [49-

107 51], and evolutionary history [48,52] of this pathway. Ecological work on the GAL pathway has

108 revealed that gene inactivation is associated with an ecological shift in Saccharomyces

109 kudriavzevii, a close relative of the species to S. cerevisiae [53]. There is also a positive

110 association between galactose metabolism ability and the flower/Ipomoea isolation environment

111 and a negative association between galactose metabolism ability and tree or insect frass isolation

112 environments [54]. While gene gain and loss in budding yeasts may play an important role in

113 ecological adaptation, variation in gene expression is also a likely contributor [55-57]. The

114 recent publication of 332 budding yeast genomes and the identification of translational selection

115 on codon usage in a majority of these species provide a unique opportunity to test for differences

116 in GAL gene expression - inferred from optimal codon usage-across ecological niches inferred

117 from recorded isolation environments [54,58-60].

118 In this study, we characterize the presence and codon optimization of the GAL pathway in 329

119 budding yeast species and identify an association between optimization in the GAL pathway and

120 two specific ecological niches. We identify a complete set of GAL genes in 210 species and 
121 evidence of physical clustering of GAL1, GAL7, and GAL10 in 150 species. Consistent with our

122 hypothesis that codon optimization is a signature of high gene expression, we find that growth

123 rate on galactose-containing medium is positively and significantly correlated with $G A L$ codon

124 optimization. In the CUG-Ser1 major clade, which contains the opportunistic human pathogen

125 Candida albicans, codon optimization in the GAL pathway is higher in species found in human-

126 associated ecological niches when compared to species associated with insect (and not human)

127 ecological niches. In the family Saccharomycetaceae, another major clade in the

128 Saccharomycotina subphylum, which contains the model species $S$. cerevisiae, we find that

129 codon optimization in the GAL pathway is higher in species isolated from dairy-associated

130 niches compared to those from alcohol-associated niches. For example, codon optimization

131 among closely related Kluyveromyces species is nearly twice as high in species isolated from

132 dairy niches as those found associated with marine or fly niches. We also used KEGG Orthology

133 (KO) annotations to find metabolic pathways with codon optimization that correlated with $G A L$

134 optimization. We identified multiple members of the thiamine biosynthesis pathway whose

135 codon optimization is not only correlated with galactose metabolism, but associated with specific

136 ecological niches. This study serves as a foundation for future high-throughput reverse ecology

137 work that uses codon optimization to link metabolic pathways with ecological niches in

138 microbes. 
bioRxiv preprint doi: https://doi.org/10.1101/2020.07.22.214635; this version posted July 24, 2020. The copyright holder for this preprint (which was not certified by peer review) is the author/funder, who has granted bioRxiv a license to display the preprint in perpetuity. It is made available under aCC-BY-NC 4.0 International license.

A

D-Galactose

Gal1

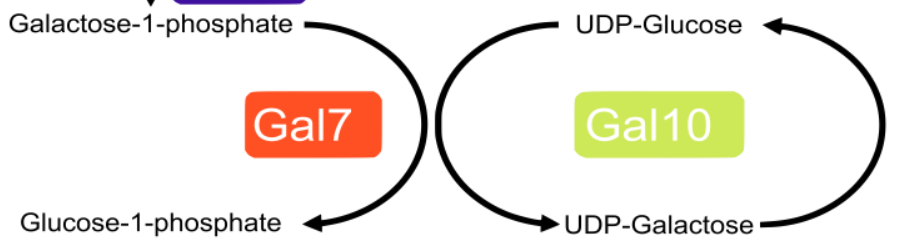

B

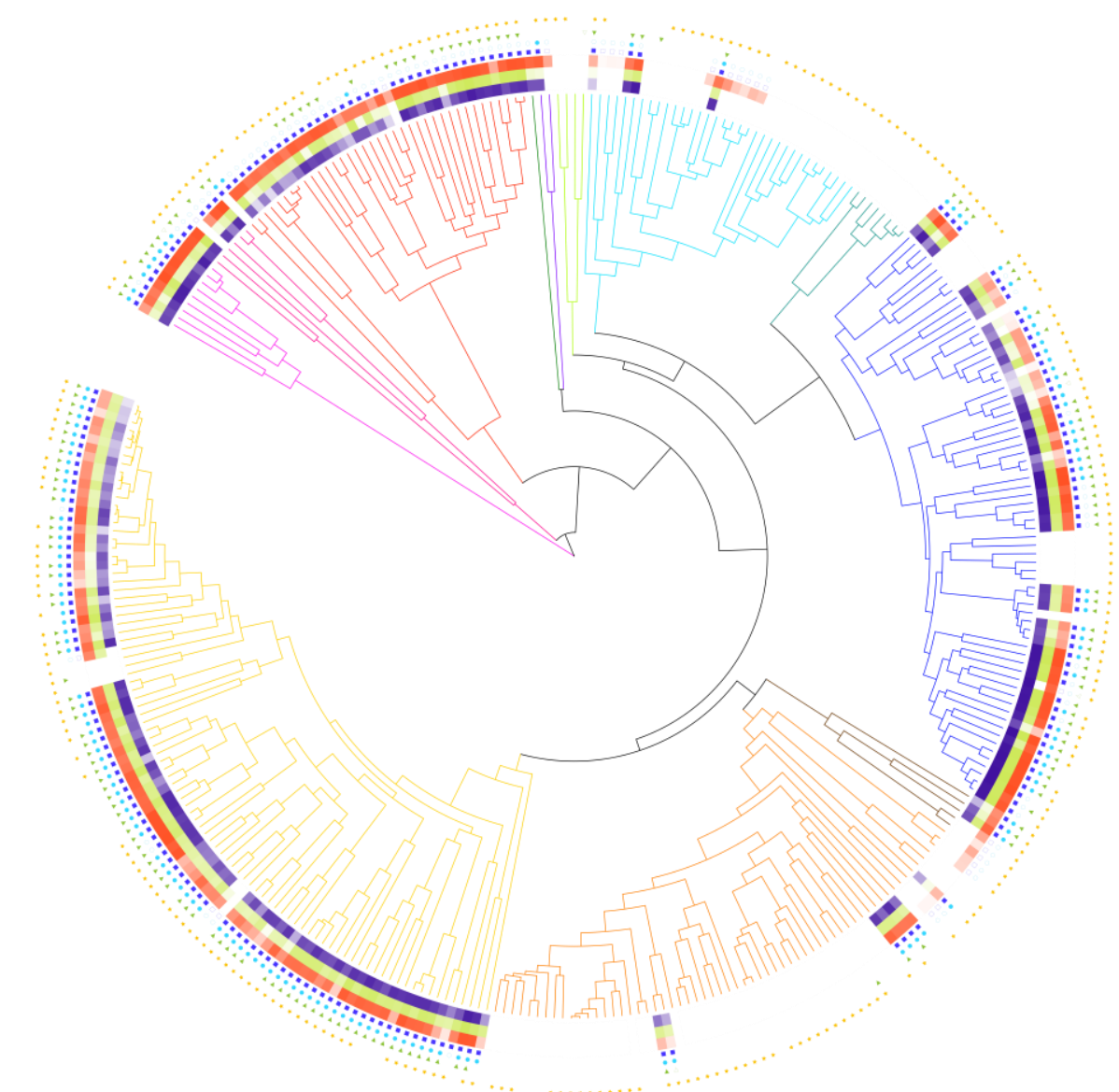

Optimal Codon Usage

0

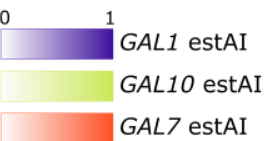

GAL7 EstAI

\section{Galactose Genes}

GAL pathway complete

GAL pathway incomplete

GAL genes clustered

GAL genes not clustered

\section{Galactose Metabolism}

A Galatose metabolism confirmed

Galatose metabolism variable

Translational Selection

on Codon Usage

s-value $>=0.5$

\section{Major Clades of Saccharomycotina}

\begin{tabular}{|c|c|c|}
\hline — Lipomycetaceae & - Sporopachydermia clade & - Saccharomycetaceae \\
\hline —Trigonopisdaceae & CUG-Ser2 clade & - CUG-Ala clade \\
\hline $\begin{array}{l}\text { Dipodascaceae/ } \\
\text { Trichomonascaea }\end{array}$ & — Phaffomycetaceae & — Pichiacea \\
\hline - Alloascoideaceae & — Saccharomycodaceae & CUG-Ser1 clade \\
\hline
\end{tabular}


Figure 1: The $G A L$ pathway and the distribution of galactose metabolism, $G A L$ genes, and preferred codon usage across the Saccharomycotina. A) The three enzymes of the $G A L$ pathway metabolize galactose into glucose-1-phosphate, which can then enter glycolysis after being converted into glucose-6-phosphate. B) Various features of galactose metabolism plotted on a phylogeny of the budding yeast subphylum Saccharomycotina; the 12 major clades of the subphylum are color-coded. The presence and codon optimization (measured by estAI) of the three $G A L$ genes are represented in the inner three rings. We did not identify any $G A L$ genes from species in the CUG-Ser2 clade or the family Saccharomycodaceae. High codon optimization (darker colors) in the $G A L$ pathway is not restricted to any one major clade. Complete and clustered occurrences of the GAL pathway (filled-in blue squares and circles respectively) are found in every other major clade examined. The ability to metabolize galactose (filled-in green triangle) was assessed either experimentally in this study or taken from the literature. In some instances where only literature data were available, there were conflicting or variable reports of galactose metabolism (5 species; empty green triangles). The majority of species in the Saccharomycotina have also been shown to have genome-wide selection on codon usage (denoted by the yellow stars)[59].

\section{METHODS}

\section{Galactose (GAL) Pathway Characterization}

159 Genomic sequence and gene annotation data were obtained from the comparative analysis of 332

160 budding yeast genomes [58] (Supplementary Table 1). Mitochondrial sequences were filtered

161 from these genomes using previously described methods[59]. Reference protein sequences for

$162 G A L$ gene annotation (approximately 40 proteins for each of the $G A L$ genes) were obtained from

163 GenBank and previous KEGG ortholog (KO) annotations [58,61]. A protein HMM profile was

164 constructed for each GAL gene and used to conduct two HMMER searches (version 3.1b2;

165 http://hmmer.org/), one on publicly available annotations and one on all possible open reading

166 frames generated using ORFfinder (version 0.4.3; https://www.ncbi.nlm.nih.gov/orffinder/). The

167 search on all possible open reading frames was done to ensure that inferences of $G A L$ gene

168 absences were not due to errors in publicly available gene annotations. The results of the two

169 searches were compared using the Perl script fasta_uniqueseqs.pl (version 1.0; 
https://www.ncbi.nlm.nih.gov/CBBresearch/Spouge/html_ncbi/html/fasta/uniqueseq.cgi).

171 Discrepancies between the two searches, which most often occurred in cases where the publicly

172 available annotation combined two nearby genes, were resolved manually. The genes GAL1 and

173 GAL3 are known ohnologs (i.e., paralogs that arose from a whole genome duplication event)

$174[62,63]$. Thus, the identity of GAL1 and GAL3 genes was inferred for Saccharomyces species by

175 phylogenetic analysis of the GAL1/3 gene tree constructed using the IQ-Tree webserver

176 (http://iqtree.cibiv.univie.ac.at/; default parameters; Supplementary Figure 1)[64-66]. Other

177 GAL1 homologs were included as there is a lack of evidence for functional divergence in other

178 lineages [51]. All reference and annotated GAL genes are available in the supplementary

179 FigShare repository. All instances where GAL1, GAL7, and GAL10 were found on the same

180 contig were considered to represent $G A L$ gene clusters.

181 Codon Optimization in the $G A L$ pathway

182 To infer gene expression in the GAL pathway, we calculated the level of codon optimization in

183 each $G A L$ gene and compared it to the genome-wide distribution of codon optimization. Codon

184 optimization of individual GAL genes was assessed by calculating the species-specific tRNA

185 adaptation index (stAI) from previously calculated species-specific codon relative adaptiveness

186 (wi) values $[59,67]$. Three species that previously failed to generate reliable wi values

187 (Martiniozyma abiesophila, Nadsonia fulvescens var. elongata, and Botryozyma

188 nematodophila)[59] were removed from all subsequent analyses. The stAI software does not take

189 into account the CUG codon reassignment in the CUG-Ser1 and CUG-Ala clades. Previous

190 analysis, however, suggests that this codon is rare [59] - the average frequency of the CUG

191 codon in species where it has been reassigned is $0.005,0.003$, and 0.006 for GAL1, GAL10, and

192 GAL7, respectively - and its influence on codon optimization calculations is not significant. 
193 The stAI for each gene was calculated by taking the geometric mean of the wi values for all the

194 codons, except the start codon. The genome-wide distribution of gene stAI values is normally

195 distributed, but the mean varies between species [59]. To compare codon optimization between

196 species, we normalized each gene's stAI value using the empirical cumulative distribution

197 function to get the percentage of all genes with stAI values lower than that of the gene of

198 interest; we call this the estAI value. For example, an estAI value of 0.4 for a given gene would

199 indicate that $40 \%$ of the genes in the genome have lower stAI values (i.e., are less optimized)

200 than the gene of interest. The estAI optimization values therefore range from 0 to 1 , with 1 being

201 the most optimized gene in the genome.

202 A total of 49 species' genomes had multiple copies of at least one $G A L$ gene. For those genomes,

203 the gene with the highest estAI value was used. For example, we identified two copies of GAL10

204 in Candida ponderosae located on different contigs with estAI values of 0.46 and 0.44 .

205 Therefore, we used the estAI value of 0.46 as the representative GAL1O value for this species.

206 The average difference between the maximum and minimum estAI for multiple copies of GAL1,

207 GAL7, and GAL10 are 0.0948, 0.0007, and 0.0125. There were 14 cases where all gene copies

208 with the highest estAI values were not found on the same contig. In 18 cases, all duplicates with

209 the highest estAI values were located on the same contig. The use of the GAL gene copy with the

210 highest estAI is supported by evidence in $S$. cerevisiae that functionally derived gene duplicates

211 have reduced codon optimization, which is likely linked to an evolutionary trajectory towards

212 novel functions [68]. 


\section{Galactose Growth Data}

216 To test the hypothesis that high levels of GAL codon optimization are associated with strong

217 growth in media where galactose is the sole carbon source, we measured galactose and glucose

218 (as a control) growth for 258 species in the laboratory. Yeast strains corresponding to the species

219 whose genomes were sequenced were obtained from the USDA Agricultural Research Service

220 (ARS) NRRL Culture Collection in Peoria, Illinois, USA or from the Fungal Biodiversity Centre

221 (CBS) Collection in the Netherlands. All strains were initially plated from freezer stocks on yeast

222 extract peptone dextrose (YPD) plates and grown for single colonies. YPD plates were stored at

$22344^{\circ} \mathrm{C}$ until the end of the experiment. To quantify growth on galactose and glucose, we set up

224 three replicates on separate weeks using different colonies for each strain. Strains were

225 inoculated into liquid YPD and grown for six days at room temperature. For each replicate,

226 strains were randomized and arrayed into a 96-well plate. The plate was then used to inoculate

227 strains into a minimal medium containing $1 \%$ D-galactose or $1 \%$ glucose, $5 \mathrm{~g} / \mathrm{L}$ ammonium

228 sulfate, and 1.7g/L Yeast Nitrogen Base (w/o amino acids, ammonium sulfate, or carbon) and

229 grown for seven days. After a week, we transferred all strains to a second 96-well plate

230 containing fresh minimal medium containing galactose or glucose.

231 To quantify the growth of each strain/species, we measured its optical density (OD units at

232 600nm) following growth in a well of a BMG LABTECH FLUOstar Omega plate reader after a

233 week at room temperature. We calculated two measures of growth, growth rate and endpoint, for

234 each species and replicate. The growth rates were calculated in $\mathrm{R}$ (x64 3.5.2) using the grofit

235 package (v 1.1.1.1) and end point, a proxy for saturation, was calculated by subtracting the $\mathrm{T}_{0}$

236 time point from the final time point for each species. We visually assessed growth on galactose

237 for all species using the growth curves we collected; a species was denoted as having the ability 
to grow on galactose if it grew in at least 2 of 3 replicates tested. Growth data, both growth rate and endpoint, were set to zero for all species that did not meet this requirement. Quantitative growth on galactose was successfully measured for a total of 258 species. Growth on galactose

241 was then computed relative to glucose to account for differences in the baseline growth rate of

242 different species due to variables, such as cell size and budding type (unipolar versus bipolar).

243 For the 71 species where new quantitative galactose growth data were unavailable, we used

244 previously published species-specific binary growth data $[54,58,60]$. Uncertain growth is

245 indicated where conflicting or variable growth was found in the literature (empty green triangles;

246 Figure 1B). Quantitative galactose growth data (normalized to glucose) were compared to

247 maximum gene codon optimization values using phylogenetically independent contrasts

248 (PIC)[69]. Data from related species are not independent observations and therefore require a

249 PIC analysis to ensure that covariation between traits is not the result of the relatedness of

250 species [69]. The PIC analysis was conducted in R using the ade4 package [70]. The species

251 Metschnikowia matae var. matae was removed from this analysis as it was a clear outlier on the

252 residual plots for a complementary PGLS analysis (Supplementary Figure 2)[71,72]. Outliers in

253 phylogenetically independent analyses occur when two closely related taxa have disparate trait

254 values, which can be identified by examining the residual plots. In this case, the taxa

255 Metschnikowia matae var. maris (yHMPu5000040795 / NRRL Y-63737 / CBS 13985) and

256 Metschnikowia matae var. matae (yHMPu5000040940 / NRRL Y-63736 / CBS 13986) are very

257 closely related, and yet the growth rate on galactose for Metschnikowia matae var. matae (1.390)

258 is nearly double that of Metschnikowia matae var. maris (0.750) and the next most closely

259 related species Metschnikowia lockheadii (0.567). 


\section{Ecological association analysis}

262 To test for associations between $G A L$ pathway codon optimization and ecological niche, we

263 obtained species-specific isolation data from multiple sources. We first tested 50 isolation

264 environments from data collated from The Yeasts: A Taxonomic Study[58,60], as recorded by

265 Opulente and coworkers [54,58]. We compared codon optimization in each of the GAL genes

266 between species isolated from a given environment versus species not isolated from that

267 environment (Supplementary Figure 3). From this analysis, we identified four general ecological

268 niches with potentially differential codon optimization: dairy-, alcohol-, insect-, and human-

269 associated ecological niches. To validate and update the data from The Yeasts, we conducted an

270 in-depth literature search for these four specific ecological niches for each of the 329 species of

271 interest using all known anamorphs and synonyms per species (see Supplementary Table 2 for

272 updated information for the ecological niches and associated references). Dairy ecological niches

273 identified included milks, butters, cheeses, and yogurts. Alcohol ecological niches identified

274 included spontaneous beer fermentation, alcohol starters, wine, ciders, kombuchas, and liquors.

275 Insect-associated ecological niches included insect guts, insect bodies, and insect frass. Human-

276 associated ecological niches were characterized as any isolation from a human, regardless of

277 pathogenicity. Additionally, we did not take into account studies where species identification

278 lacked genetic data and relied solely on phenotypic and assimilation data, because these

279 identifications have been shown to be potentially unreliable [73-75]. For example, the only

280 evidence that the species Candida castellii is associated with dairy niches comes from a single

281 identification in a fermented milk product using only metabolic chacterization [76]. Therefore,

282 C. castellii was not considered associated with dairy niches. 
284 To test for significant differences in GAL optimization between ecological niches, we first

285 filtered the species set to retain only those that contain all three $G A L$ genes (210 species) and that

286 were previously shown to exhibit genome-wide selection on codon usage (266 species; s-value

$287>=0.5)[59]$; thus, the total number of species tested was 170. We then compared levels of $G A L$

288 codon optimization between ecological niches using the Wilcoxon rank sum test in R [77].

\section{Evolutionary rate analysis}

290 To examine variation in the evolutionary rates among $G A L$ genes, we used the maximum

291 likelihood software PAML (version 4.9)[78,79]. Specifically, we examined the rates of

292 synonymous changes in the Kluyveromyces species using the free-ratios model that allows for a

293 different rate of evolution along each branch. The species tree was used as the backbone tree, and

294 nucleotide sequences were aligned using the codon aware software TranslatorX

295 (http://translatorx.co.uk/)[80].

\section{Identification of additional metabolic pathways whose codon usage correlates with $G A L$}

\section{7 optimization}

298 To identify additional pathways that exhibit the same codon optimization trends between

299 ecological niches as the GAL pathway, we tested whether the optimization of KEGG orthologs

300 (KOs) was correlated with that of the GAL genes. KO annotations were previously generated for

301 all species [58]. We started with the 266 genomes with evidence of translational selection on

302 codon usage and identified 2,573 KOs present in 100 or more of those species. We then

303 conducted a PIC analysis between the optimization of the GAL genes and each of the KOs across

304 the species. P-values were adjusted to account for the total number of KOs tested using a

305 Bonferroni correction (Supplementary Table 3). Based on the results of the PIC analysis, we 
further investigated the correlation between the thiamine biosynthesis pathway and the $G A L$

307 pathway. To ensure we were not missing any members of the thiamine biosynthesis pathway, we

308 annotated the entire pathway using the same method used for annotation of the GAL genes. We

309 then re-ran the PIC analysis with the curated thiamine gene set.

RESULTS \& DISCUSSION

Variable GAL pathway and codon optimization across the Saccharomycotina

312 To examine variation in GAL codon optimization across the subphylum, we first examined

313 whether GAL genes were present in each of the 329 genomes. Across the Saccharomycotina, we

314 annotated 742 GAL genes (265, 256, and 221 annotations for GAL1, GAL10, and GAL7,

315 respectively) in a total of 233 species (Supplementary Table 1 and FigShare Repository). The

316 complete $G A L$ enzymatic pathway (i.e., GAL1, GAL10, and GAL7) was identified in 210 species,

317 of which 149 had evidence of GAL gene clustering. We cannot, however, rule out clustering of

318 the $G A L$ genes in the remaining 61 species as some of the annotations were at the ends of the

319 contigs.

320 There were some discrepancies between galactose growth data and GAL gene presence data.

321 Three species where galactose growth was experimentally observed lacked all three GAL genes:

322 Ogataea methanolica, Wickerhamomyces sp.YB-2243, and Candida heveicola. The growth rates

323 for these species are $0.129,0.339$, and 0.211 for $O$. methanolica, Wickerhamomyces sp., and $C$.

324 heveicola. The low growth rates ( $7^{\text {th }}$ and $3^{\text {rd }}$ lowest overall) of $O$. methanolica and $C$. heveicola

325 suggest these species may be utilizing trace amounts of other nutrients present in the medium.

326 Finally, there were 26 species with a complete $G A L$ gene cluster where no growth on galactose

327 has been reported. This may represent a loss of pathway induction in these species or an inability 
to induce growth in the specific experimental conditions tested, as observed previously in the genus Lachancea [81]. Inactivation of the GAL pathway has also occurred multiple times in

330 budding yeasts [48,53], and some of these taxa could be in the early stages of pathway

331 inactivation.

332 Codon optimization in the GAL pathway, measured by estAI, varied greatly across the

333 Saccharomycotina (Figure 1B.) The estAI values ranged from 0.02 (or greater than only $2 \%$ of

334 the genes in the genome) in GAL7 from Lachancea fantastica nom. nud. to 0.99 (or greater than

$33599 \%$ of the genes in the genome) in GAL1 from Kazachstania bromeliacearum. To determine if

336 there is an association between codon optimization and the ability to grow on galactose, we

337 compared optimization in the GAL pathway between species that are able and unable to grow on

338 galactose. We found that species without evidence for growth on galactose had significantly

339 lower ( $\mathrm{p}<0.05$ ) codon optimization in GAL1 and GAL7 (Supplementary Figure 4). This

340 correlation is consistent with a relaxation of selective pressures in non-functional pathways [82-

$34184]$ and previous work has identified multiple parallel inactivation events of the GAL pathway in

342 budding yeasts [53]. The GAL pathway may have alternative roles in cell function that are not

343 associated with growth on galactose and may have not experienced the same selective pressures.

344 For example, in Candida albicans, GAL10 has been shown to be involved in cell integrity [85].

345 Finally, the GAL pathway may have an alternative induction system in these species. For

346 example, the fission yeast Schizosaccharomyces pombe (not a member of the Saccharomycotina)

347 has a complete $G A L$ cluster but is unable to grow on galactose. Mutants of $S$. pombe, however,

348 have been isolated that constitutively express the $G A L$ genes and can grow on galactose [86]. 


\section{$G A L$ codon optimization is correlated with growth rate on galactose}

351 Strong translational selection on codon usage is correlated with highly expressed genes in

352 diverse organisms [34,35,37,87-91]. Therefore, we hypothesized that high levels of codon

353 optimization in the GAL pathway reflect high levels of GAL gene expression and ultimately high

354 growth rates on galactose. To test this hypothesis, we measured growth rate on galactose relative

355 to glucose. We found a significant positive correlation between growth rate on galactose-

356 containing medium and codon optimization in the $G A L$ pathway of genomes that have

357 experienced translational selection on codon usage $(\mathrm{N}$ species $=94$, linear regression of PIC

358 values; p-values of $0.005,0.012$, and $3.207 \mathrm{e}^{-9}$ for $G A L 1, G A L 10$, and GAL7, respectively; Figure

359 2). Codon optimization of GAL7 showed the strongest correlation with growth rate (Figure 2C),

360 which may reflect the gene's function; GAL7 encodes for the enzyme that metabolizes galactose-

361 1-phosphate, a toxic intermediate $[92,93]$ whose accumulation has been shown to reduce growth

362 rate in S. cerevisiae [93]. Furthermore, the correlation between GAL7 optimization and growth

363 rate on galactose remained strong when analyzed independently in both the Saccharomycetaceae

364 (29 species) and in the CUG-Ser1 clade (47 species; Supplementary Figure 5), the two largest

365 clades sampled. The GAL1 and GAL10 genes were both significantly positively associated with

366 growth rate in galactose in the Saccharomycetaceae, but not in the CUG-Ser1 clade

367 (Supplementary Figure 5). This contrast may reflect the different regulatory mechanisms

368 involved in galactose assimilation in the two major clades - tight control via a regulatory switch

369 in the Saccharomycetaceae versus leaky expression in CUG-Ser1 [49,50]. We also tested the

370 correlation between growth rate on galactose containing medium and the PGM1 and PGM2

371 genes that encode phosphoglucomutases which converts $\backslash$ glucose-1-phosphate (Figure 1) to

372 glucose-6-phosphate. There was no correlation between optimization in PGM1 or 2 and growth 
373 on galactose containing medium (Supplementary Figure 6). Collectively, our findings support

374 the hypothesis that codon optimization is the result of selection on codon usage in species with

375 high GAL gene expression.
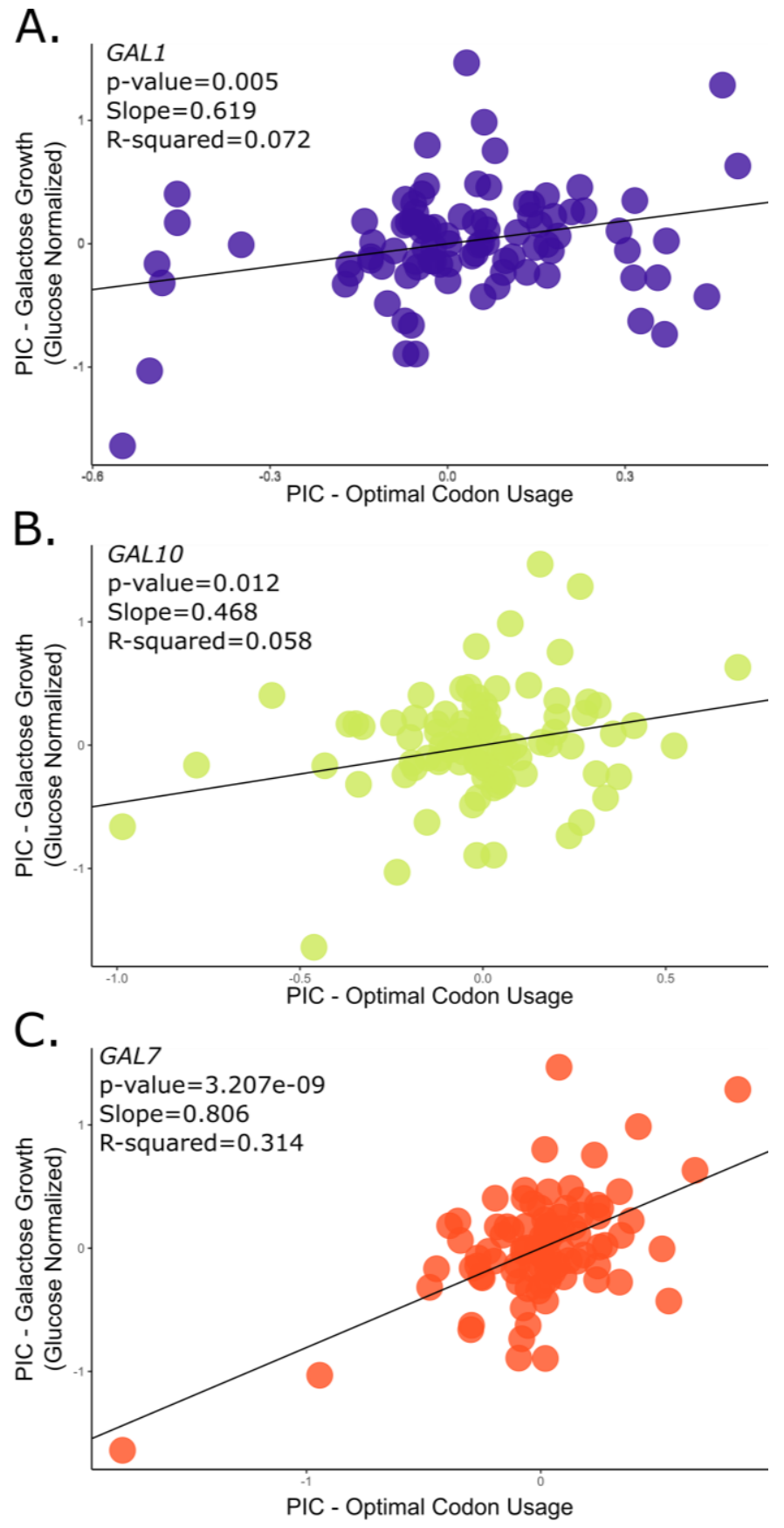

Figure 2: Codon optimization in the $G A L$ pathway is positively and significantly correlated with growth rate on galactose. Phylogenetically independent contrasts (PIC) analyses of galactose growth (Y axis) versus $G A L$ gene optimal codon usage ( $\mathrm{X}$ axis). There is a significant and positive correlation between the PIC values for codon optimization and galactose growth in GAL1 (A), GAL10 (B), and GAL7 (C). The best fit and strongest correlation is between growth on galactose and optimization in GAL7 (C). The analyses included 94 species with a growth rate on galactose greater than 0 , a complete $G A L$ cluster, and evidence of genomewide translational selection on codon usage. One species, Metschnikowia matae var. matae, was removed as an obvious outlier based on residual analysis. 


\section{$377 G A L$ codon optimization is associated with specific ecological niches}

378 We further hypothesized that adaptation to specific ecological niches is associated with increased

379 expression of the GAL pathway. Based on preliminary tests across 50 previously characterized

380 ecological niches [54,60] for 114 species, we conducted an extensive literature search for the

381 four ecological niches of interest - dairy, alcohol, human, insect - to maximize the number of

382 species with ecological information. We uncovered two examples of niche-specific codon

383 optimization (Figure 3): in the CUG-Ser1 clade, we found that GAL gene optimization was

384 significantly higher in species that have been isolated from human-associated ecological niches

385 versus those that have been isolated from insect-associated niches; and in the

386 Saccharomycetaceae, we found $G A L$ gene optimization was significantly higher in species

387 isolated only from dairy-associated niches compared to species isolated only from alcohol-

388 associated niches.

CUG-Ser1 clade: Among CUG-Ser1 species that exhibit high genome-wide evidence of

390 translational selection on codon usage (s-value $\geq 0.5$ ), we found that $G A L$ gene optimization was

391 significantly higher $(\mathrm{p}<0.05)$ in species from human-associated ecological niches or human- and

392 insect-associated niches versus those that have been isolated from insect-associated niches only

393 (57 species; Figure 3A). Only two species were found in human-associated niches and not insect-

394 associated niches, Debaryomyces subglobosus and Cephaloascus fragrans; thus, we combined

395 the human-associated species with the human- and insect-associated species into one group for

396 subsequent analyses. Recent work has shown that many opportunistically pathogenic budding

397 yeasts are likely to be associated with both environmental and human niches [94]. The 13 CUG-

398 Ser1 species isolated from humans with genome-wide evidence of selection on codon usage had

399 a mean optimization of $0.74,0.76$, and 0.69 for $G A L 1, G A L 10$, and GAL7, respectively. 
We also found that GAL1, GAL10, and GAL7 optimization was significantly higher (Wilcoxon rank sum test $\mathrm{p}$-values of $0.035,0.014$ and 0.003 , respectively) in species from human-associated ecological niches than insect-associated niches only, irrespective of genome-wide evidence of translational selection (88 species). For example, the major human pathogen Candida albicans does not have genome-wide evidence for high levels of translational selection but has a very high GAL10 codon optimization (estAI $=0.86)$. While $C$. albicans may not have evidence of genomewide selection on codon optimization, a previous analysis suggests that at least $17 \%$ of genes in the $C$. albicans genome have likely experienced selection on codon usage [59]. > 0.8) include Candida dubliniensis [95], Meyerozyma caribbica [96], Candida tropicalis [97],

410 Meyerozyma guilliermondii [98], and Clavispora lusitaniae [99]. The optimization of GAL10 in

411 human pathogenic species is consistent with findings that GAL10 expression is upregulated

412 during $C$. albicans growth in the mammalian intestinal track [100]. Furthermore, GAL10 in $C$.

413 albicans is required for cell-wall integrity, resistance to oxidative stress, and other virulence-

414 related traits, even in the absence of galactose [85]. This suggests that GAL10 may play an

415 additional role, outside of galactose metabolism, in the CUG-Ser1 clade.

416 Interestingly, the highest GAL10 optimization (average estAI $=0.93$ ) in the CUG-Ser1 clade is

417 found in Spathaspora species. While many Spathaspora species have been isolated from insects,

418 four of the five species studied here (Sp. girioi, Sp. hagerdaliae, Sp. gorwiae, and Sp.

419 arborariae) have been isolated only from rotting wood [101,102]. This observation is

420 particularly interesting given the hypothesis that some features of saprophytic fungi, such as

421 Aspergillus fumigatus and Cryptococcus spp., enable or predispose them to colonize human hosts 
423 have recently been associated with soil [94].

\section{A. CUG-Ser1 Clade}

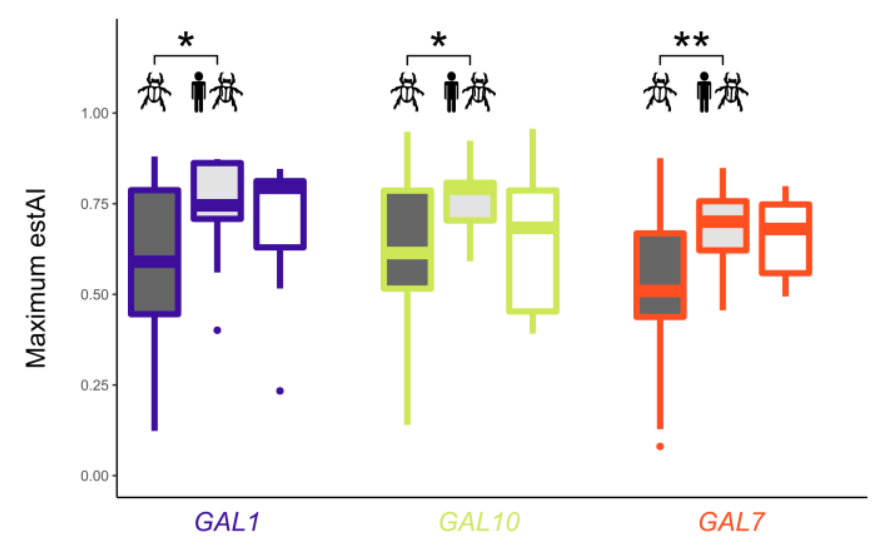

B. Saccharomycetaceae

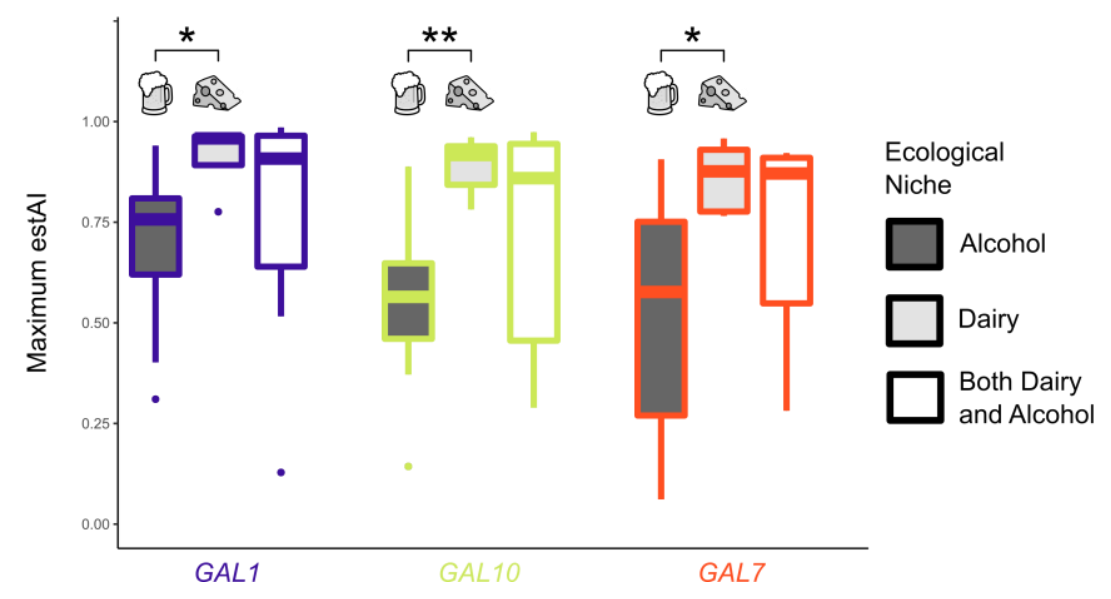

Figure 3 - Codon optimization in the GAL pathway is correlated with specific ecological niches in two different major clades of budding yeasts. P-values less than 0.01 are indicated with ** and less than 0.05 with *. A) In the CUG-Ser1 clade, species associated with a human niche or human and insect niches (13 species) have significantly higher codon usage optimization values in all GAL genes (p-values of 0.022, 0.028, and 0.006 for GAL1, GAL10, and $G A L 7$, respectively) when compared to species that are associated with insect niches but not human niches (44 species). Only 11 species were not associated with either human or insect niches. B) In the Saccharomycetaceae, species associated with only dairy niches (5 species) have significantly higher codon usage optimization values in all of the $G A L$ genes (p-values of 0.010 , 0.002 , and 0.014 for GAL1, GAL10, and GAL7, respectively) versus species associated with only alcohol niches (14 species). A total of 9 species are associated with both dairy and alcohol niches. 
Saccharomycetaceae: Among Saccharomycetaceae species, we found that GAL optimization is

438 significantly higher $(\mathrm{p}<0.05)$ in those that have been isolated only from dairy-associated niches

439 compared to species isolated only from alcohol-associated niches (19 species; Figure 3B.) Only

440 one species isolated from either dairy or alcohol, namely the alcohol-associated Lachancea

441 thermotolerans, did not have evidence of genome-wide translational selection on codon usage.

442 The four species isolated only from dairy-associated niches (Kluyveromyces lactis,

443 Naumovozyma dairenensis, Vanderwaltozyma polyspora, and Kazachstania turicensis) have

444 mean codon optimization values of $0.90,0.88$, and 0.84 for GAL1, GAL10, and GAL7,

445 respectively. The ten species that are only from alcohol-associated niches (Supplementary table

446 2) have mean codon optimization values of $0.73,0.61$, and 0.59 for GAL1, GAL10, and GAL7,

447 respectively. In many dairy environments, there are large microbial communities that often

448 consist of lactic acid bacteria that convert lactose into glucose and galactose, which can

449 subsequently be used in the $G A L$ pathway $[105,106]$. The natural presence of galactose in dairy-

450 associated environments is the likely driver of $G A L$ codon optimization.

451 Species found in both dairy- and alcohol-associated niches have a range of optimization values

452 that generally encompasses the values observed for species from dairy- or alcohol-only niches. It

453 is likely that this group (associated with both dairy and alcohol niches) contains species or

454 populations that are better adapted to one niche than the other. It is not possible, however, based

455 on current literature to disentangle these two categories. For example, the species Kluyveromyces

456 marxianus has been isolated from chica beer [107], cider [108], kombucha [109], and mezcal

457 liquor [110]. However, K. marxianus is a well-known "dairy-yeast" frequently found in both

458 natural [111,112] and industrial dairy products [113]. Codon optimization of the GAL enzymatic

459 pathway is also very high in $K$. marxianus with an average estAI of 0.92 . We hypothesize that 
460 the high GAL codon optimization in $K$. marxianus is a result of its association with dairy and

461 with the ability of $K$. marxianus to metabolize lactose into glucose and galactose [44]. There are

462 two species that are associated with both dairy and alcohol niches whose $G A L$ codon

463 optimization values are higher than the maximum value observed in alcohol-only species-

464 Naumovozyma castellii and Kazachstania unispora. Based on this we hypothesize that these

465 species are well adapted to dairy-associated environments.

\section{Differential $G A L$ pathway optimization in Kluyveromyces}

467 The genus Kluyveromyces provides an example of how codon optimization varies between

468 closely related species that differ in their ecological niches (Figure 4). Two of the four species in

469 this clade have not been isolated from either dairy or alcohol; Kluyveromyces aestuarii has been

470 isolated from marine mud and seawater while Kluyveromyces dobzhanskii has been isolated from

471 flies, plants, and mushrooms [60]. Of the four species represented here, only K. dobzhanskii is

472 not known to metabolize lactose into glucose and galactose [60]. While all four species are

473 capable of growing on galactose, $G A L$ gene codon optimization is much higher in the two

474 species with dairy-associated ecological niches, Kluyveromyces lactis and Kluyveromyces

475 marxianus (Figure 4A.). Codon optimization for $G A L$ genes is greater than $75 \%$ of the genome

476 (estAI > 0.75) for K. lactis and K. marxianus. In K. marxianus, the optimization of GAL1 and

477 GAL10 (estAI 0.93 and 0.94) is nearly that of the average ribosomal gene (estAI 0.99; Figure

478 4B). Ribosomal genes, which are among the most highly expressed genes in the genome, are

479 known to be highly optimized in a broad range of species [114]. In contrast, optimization values

480 for GAL genes in $K$. aestuarii and $K$. dobzhanskii are nearer to the mean (mean estAI values of

4810.63 and 0.46, respectively; Figure 4B). 
A.

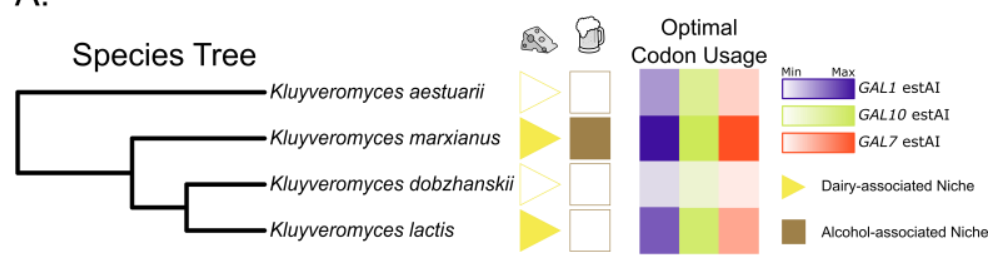

B.
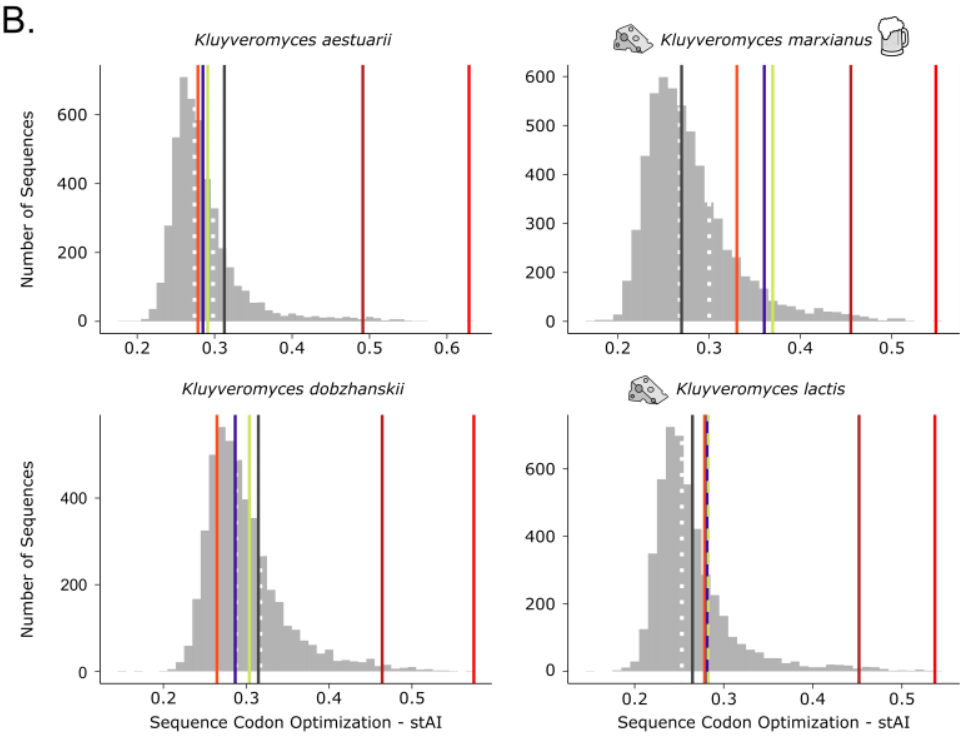

|gAL1 |GAL7 || GAL10 |VPS4 |Maximum Ribosome |Average Ribosome C.

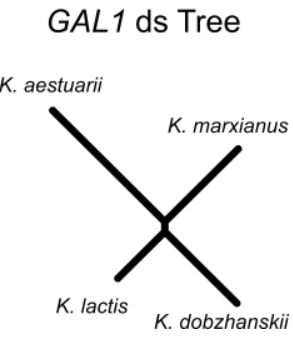

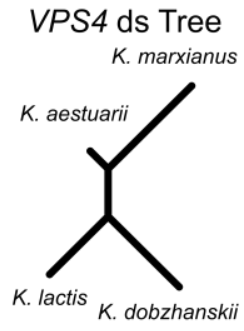

Figure 4 - Closely related Kluyveromyces species exhibit differential codon optimization in the $G A L$ pathway associated with isolation from dairy environments. All four Kluyveromyces species were shown experimentally to metabolize galactose. A) Species phylogeny of four closely related Kluyveromyces species. $K$. marxianus and $K$. lactis are both associated with dairy niches and have high codon optimization values in their $G A L$ pathway genes. In contrast, $K$. aestuarii is associated with marine mud, and $K$. dobzhanskii is associated with flies. B) The genome-wide distribution of codon optimization (stAI) values for the four Kluyveromyces species included in this study. The $50^{\text {th }}$ and $75^{\text {th }}$ percentiles are shown with white dashed lines. In the two species associated with dairy niches, the codon optimization for all three $G A L$ genes falls in the top $25^{\text {th }}$ percentile. In the two species not associated with dairy, the GAL genes fall below the top $25^{\text {th }}$ percentile. The gene VPS4 (encoding a protein

involved in vacuolar protein sorting) is a non-metabolic gene with intermediate codon optimization value across budding yeasts. Genes encoding ribosomal proteins are well established to rank among the most highly optimized genes within a genome. C) The unrooted trees show the estimated rate of synonymous substitutions in the GAL1 and VPS4 genes along these lineages. The long branch in $K$. aestuarii for the GAL1 tree suggests a relaxation of selection on synonymous sites in this lineage. 
the rate of synonymous site evolution using PAML in the GAL genes and VPS4, a randomly chosen KEGG ortholog annotated in all 4 species. In each of the GAL genes, the branch length for $K$. aestuarii was the longest, and is at least double in length relative to the other branches in the GAL7 and GAL10 gene trees (Figure 4C; Supplementary Figure 7). The branch lengths of $K$. dobzhanskii were similar to those of $K$. marxianus in the trees of all three GAL genes. This

491 pattern was not seen in the randomly chosen VPS4 gene. This result suggests that relaxed 492 selection on the GAL genes may exist in $K$. aestuarii, but not $K$. dobzhanskii, or that the 493 relaxation may have persisted longer in $K$. aestuarii. Increased sampling in this clade would 494 improve our understanding of the selective forces at work. adaptation to an ecological niche [54,115]. To identify additional pathways associated with

498 galactose optimization, we tested whether levels of codon optimization in $G A L$ genes were 499 significantly correlated with levels of codon optimization in other KEGG orthologs (KOs). We identified 78 / 2,572 KOs with a significant positive or negative association with $G A L$ optimization (PIC, multiple test corrected p-value <0.05; Supplementary Table 3). One of the strongest positive associations ( $8^{\text {th }}$ smallest p-value in GAL10 out of $28 \mathrm{KOs}$ with significant

503 positive associations) was with THI6 (KO K14154), a member of the thiamine biosynthesis 504 pathway (Figure 5A and B). We expanded our analysis to the two branches of the thiamine 505 biosynthesis pathway present in the budding yeast subphylum that converge on THI6 (Figure

$5065 \mathrm{C})$. On the branch of the thiamine biosynthetic pathway that begins with the substrates 507 pyridoxal 5'phosphate and L-histidine, we found significantly $(\mathrm{p}<0.05)$ correlated codon 508 optimizations between the THI20/THI21/THI22 gene family and the GAL genes GAL1 and 
509 GAL10 (Figure 5C). In the other branch of the pathway, codon optimization in THI4 is only

510 correlated with GAL10 (Figure 5C). Among genes involved in thiamine biosynthesis, the

511 strongest association with the GAL pathway was seen in THI6 where there was a significant

512 positive association with optimization in all three $G A L$ genes (Figure 5B). The positive

513 correlation seen using PIC suggests that this association does not reflect phylogenetic constraint

514 but adaptation.

515 Support for the notion that ecological adaptation explains the correlation between the thiamine

516 biosynthesis and GAL pathways can be found in both major clades examined. Within the CUG-

517 Ser1 clade, there is a significantly higher $(\mathrm{p}<0.05)$ THI6 codon optimization in species associated

518 with either human or insect ecological niches when compared to species only isolated from

519 insect ecological niches (Figure 5D). The difference in THI6 codon optimization is even more

520 significant $(\mathrm{p}<0.001)$ in the Saccharomycetaceae where THI6 codon optimization is higher in

521 species only associated with dairy ecological niches and not alcohol ecological niches (Figure

522 5E). Many lactic acid bacteria found in dairy environments, such as Lactobacillus brevis, require

523 extracellular thiamine [116]. One possible model is that, in dairy communities containing lactic

524 acid bacteria and yeasts, stiff extracellular competition for thiamine may boost the expression of

525 thiamine biosynthesis genes in these yeasts. Alternatively, the thiamine biosynthesis and

526 galactose metabolism pathways may be connected by metabolic intermediates [117]. It is

527 possible that both a biochemical and ecological explanation underlie the correlation between

528 codon optimization in the GAL and thiamine biosynthesis pathways. 
A.

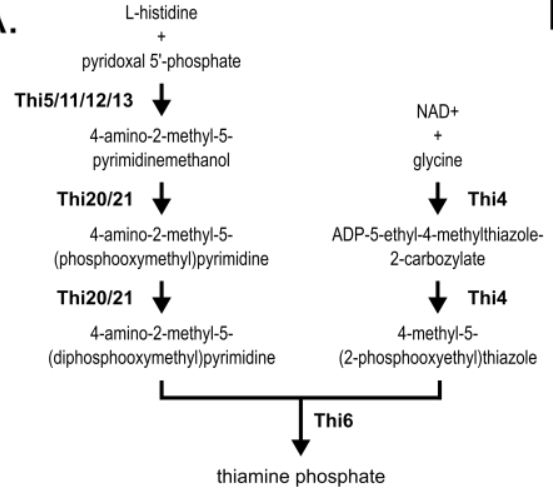

C.

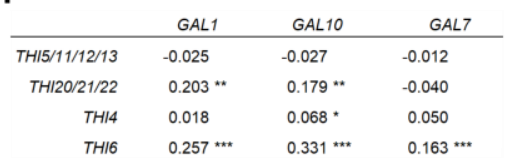

D.

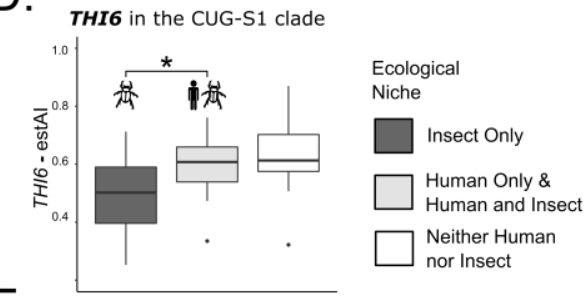

E.

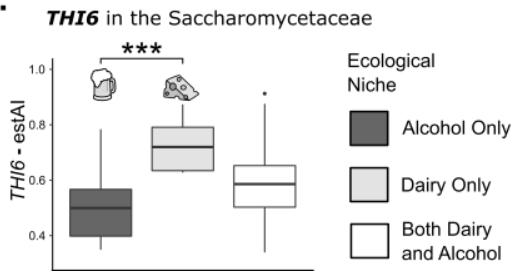

B.
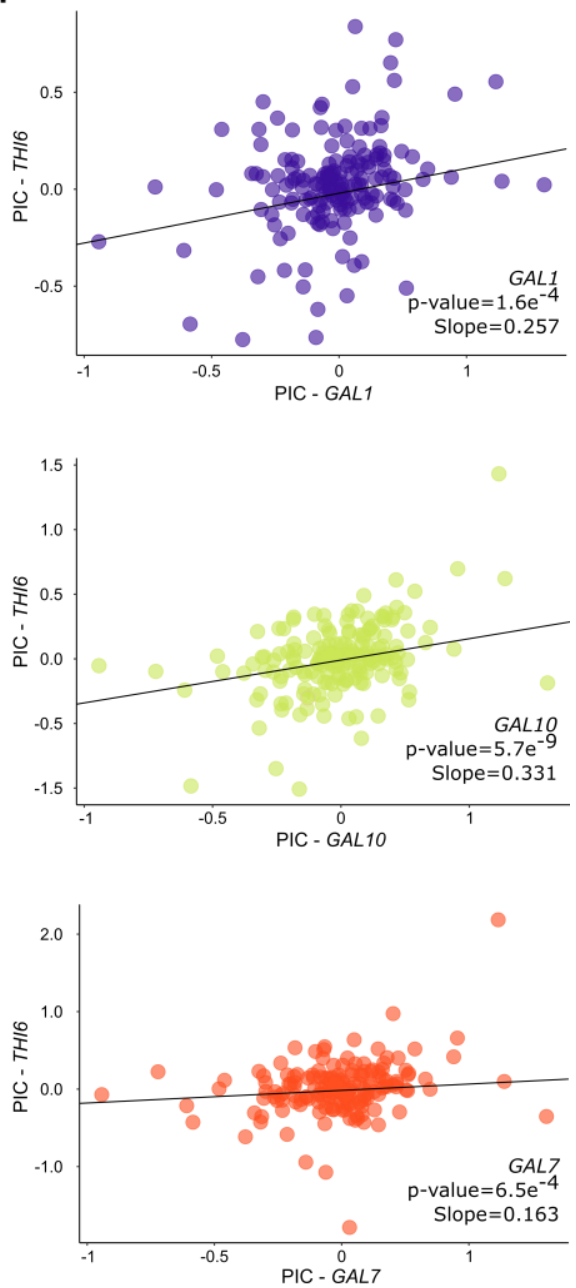

Figure 5 - Codon optimization in the $G A L$ pathway is positively and significantly correlated with optimization of multiple thiamine biosynthesis proteins. A) The two branches of the thiamine biosynthesis pathways present in budding yeasts converge on THI6. B) The PIC correlation between codon optimization in the GAL genes and THI6 in species with evidence of genome-wide translational selection on codon usage (s-value $>=0.5$ ). The strongest correlation is between GAL10 and THI6 (182 species), followed by GAL1 (168 species), and GAL7 (170 species). C) Optimization in GAL10 is also correlated with optimization of the THI20/THI21/THI22 gene family and THI4- these genes encode the enzymes upstream of THI6. Optimization in GAL1 is additionally correlated with THI20/THI21/THI22 optimization. There is no correlation between optimization in the THI5/THI11/THI12/THI13 gene family and any GAL genes. D) Optimization in THI6 is significantly greater in CUG-Ser1 clade species associated with human or human and insect ecological niches (14 species) when compared to species associated only with insect ecological niches ( 48 species) ( $p$-value $=0.011$ ). Twelve species were not associated with either human or insect ecological niches. E) Optimization in THI6 is significantly higher in Saccharomycetaceae species associated with dairy ecological niches (6 species) versus those associated with alcohol ecological niches ( 16 species) $\left(p\right.$-value $\left.=1.9 \mathrm{e}^{-4}\right)$. Ten species with THI6 are associated with both ecological niches, and 33 species are not associated with either environment. 


\section{CONCLUSIONS}

549 Here we use reverse ecology to connect genotype (codon optimization) with phenotype (growth

550 rate on galactose) and ecology (isolation environment) across an entire evolutionary lineage

551 (budding yeasts). By studying a well-known metabolic pathway in a diverse microbial

552 subphylum, we provide a proof of concept for the utility of codon optimization as a genomic

553 feature for reverse ecology. Our discovery of optimization in the GAL pathway in dairy-

554 associated Saccharomycetaceae and human-associated CUG-Ser1 yeasts is consistent with the

555 known functional roles of the enzymes in the pathway. The complete $G A L$ pathway metabolizes

556 lactose, a component of dairy environments, into usable energy [118]. The GAL10 gene is

557 associated with phenotypes associated with human colonization in CUG-Ser1 yeasts [85].

558 Similarly, in the Kluyveromyces species found on dairy-associated niches that are able to

559 metabolize lactose into glucose and galactose, there is high optimization in this pathway

560 compared to closely related species not associated with dairy. Interestingly, examination of

561 codon optimization in the gene sets of the four Kluyveromyces species studied here would have

562 identified at least $K$. marxianus as a potential dairy-associated yeast, even in the absence of any

563 knowledge about its isolation environments. Thus, genome-wide examination of codon

564 optimization in fungal, and more generally microbial, species can generate specific hypotheses

565 about metabolic ecology in species for which ecological data are lacking. These results are

566 especially promising as this method can be applied directly to genomic data — which is the only

567 source of information for microbial dark matter known only from DNA [119]. Finally, using an

568 unbiased approach, we identified a strong correlation between optimization in the thiamine

569 biosynthesis pathway and the GAL pathway. This novel finding suggests that codon optimization 
bioRxiv preprint doi: https://doi.org/10.1101/2020.07.22.214635; this version posted July 24, 2020. The copyright holder for this preprint (which was not certified by peer review) is the author/funder, who has granted bioRxiv a license to display the preprint in perpetuity. It is made available under aCC-BY-NC 4.0 International license.

570 may also be useful for identifying co-regulated or correlated pathways in microbial, including

571 fungal, species. 


\section{Acknowledgements}

573 We thank the members of the Rokas and Hittinger labs for helpful discussions.

\section{Data availability}

575 All analyses were done on publicly available and published genome assemblies and annotations.

576 The codon optimization values were obtained from the figshare repository from LaBella et al.

5772019 (https://doi.org/10.6084/m9.figshare.c.4498292.v1). Additional sequence data generated in

578 this project, including the reference and annotated gene sequences, are stored in the figshare

579 repository associated with this manuscript and will be made publicly available upon acceptance

580 to a peer-reviewed journal. Reviewers can access the figshare repository through the private

581 link:. All other information and data generated are available in the supplementary files.

\section{$582 \quad$ Literature Cited}

583 1. Savolainen O, Lascoux M, Merilä J. Ecological genomics of local adaptation. Nat. Rev. Genet. 5842013.

585 2. Hoekstra HE, Krenz JG, Nachman MW. Local adaptation in the rock pocket mouse

586 (Chaetodipus intermedius): Natural selection and phylogenetic history of populations. Heredity

587 (Edinb). 2005;

588 3. Barrett RDH, Rogers SM, Schluter D. Natural selection on a major armor gene in threespine 589 stickleback. Science (80- ). 2008;

590 4. Abzhanov A, Protas M, Grant BR, Grant PR, Tabin CJ. Bmp4 and morphological variation of 591 beaks in Darwin's finches. Science (80- ). 2004; 
593 and evolution of elongated beak morphology in Darwin's finches. Nature. 2006;

594 6. Grant PR. Ecology and evolution of Darwin’s finches. Ecol. Evol. Darwin’s Finches. 2017.

595 7. Daborn PJ, Yen JL, Bogwitz MR, Le Goff G, Feil E, Jeffers S, et al. A single P450 allele

596 associated with insecticide resistance in Drosophila. Science (80- ). 2002;

597 8. Steiner CC, Weber JN, Hoekstra HE. Adaptive variation in beach mice produced by two

598 interacting pigmentation genes. PLoS Biol. 2007;

599 9. Zhou J. Predictive microbial ecology. Microb. Biotechnol. 2009.

600 10. Levy R, Borenstein E. Reverse ecology: From systems to environments and back. Adv Exp

601 Med Biol. 2012;

602 11. Li YF, Costello JC, Holloway AK, Hahn MW. "Reverse ecology" and the power of 603 population genomics. Evolution (N Y). 2008;

604 12. Retchless AC, Lawrence JG. Ecological adaptation in bacteria: Speciation driven by codon 605 selection. Mol Biol Evol. 2012.

606 13. Levy R, Borenstein E. Metagenomic systems biology and metabolic modeling of the human 607 microbiome: From species composition to community assembly rules. Gut Microbes. 2014;

608 14. Sauer DB, Wang DN, Valencia A. Predicting the optimal growth temperatures of prokaryotes 609 using only genome derived features. Bioinformatics. 2019;

610 15. Ellison CE, Hall C, Kowbel D, Welch J, Brem RB, Glass NL, et al. Population genomics and 
611 local adaptation in wild isolates of a model microbial eukaryote. Proc Natl Acad Sci U S A.

$612 \quad 2011 ;$

613 16. Borenstein E, Kupiec M, Feldman MW, Ruppin E. Large-scale reconstruction and

614 phylogenetic analysis of metabolic environments. Proc Natl Acad Sci U S A. 2008;

615 17. Cao Y, Wang Y, Zheng X, Li F, Bo X. RevEcoR: An R package for the reverse ecology

616 analysis of microbiomes. BMC Bioinformatics. 2016;

617 18. Carr R, Borenstein E. NetSeed: A network-based reverse-ecology tool for calculating the

618 metabolic interface of an organism with its environment. Bioinformatics. 2012;

619 19. Ikemura T. Correlation between the abundance of Escherichia coli transfer RNAs and the

620 occurrence of the respective codons in its protein genes. J Mol Biol. 1981;

621 20. Thomas LK, Dix DB, Thompson RC. Codon choice and gene expression: Synonymous

622 codons differ in their ability to direct aminoacylated-transfer RNA binding to ribosomes in vitro.

623 Proc Natl Acad Sci U S A. 1988;

624 21. Gouy M, Gautier C. Codon usage in bacteria: Correlation with gene expressivity. Nucleic

625 Acids Res. 1982;

626 22. López-Maury L, Marguerat S, Bähler J. Tuning gene expression to changing environments:

627 From rapid responses to evolutionary adaptation. Nat. Rev. Genet. 2008.

628 23. Goldspink G. Adaptation of fish to different environmental temperature by qualitative and 629 quantitative changes in gene expression. J Therm Biol. 1995;

630 24. Xu Q, Zhu C, Fan Y, Song Z, Xing S, Liu W, et al. Population transcriptomics uncovers the 
631 regulation of gene expression variation in adaptation to changing environment. Sci Rep. 2016;

632 25. Fay JC, McCullough HL, Sniegowski PD, Eisen MB. Population genetic variation in gene

633 expression is associated with phenotypic variation in Saccharomyces cerevisiae. Genome Biol.

634 2004;

635 26. Rocha EPC. Codon usage bias from tRNA's point of view: Redundancy, specialization, and

636 efficient decoding for translation optimization. Genome Res. 2004;

637 27. Chevance FFV, Le Guyon S, Hughes KT. The Effects of Codon Context on In Vivo

638 Translation Speed. PLoS Genet. 2014;

639 28. Stoletzki N, Eyre-Walker A. Synonymous codon usage in Escherichia coli: Selection for

640 translational accuracy. Mol Biol Evol. 2007;

641 29. Tuller T, Waldman YY, Kupiec M, Ruppin E. Translation efficiency is determined by both

642 codon bias and folding energy. Proc Natl Acad Sci U S A. 2010;

643 30. Brule CE, Grayhack EJ. Synonymous Codons: Choose Wisely for Expression. Trends Genet. 6442017.

645 31. Presnyak V, Alhusaini N, Chen YH, Martin S, Morris N, Kline N, et al. Codon optimality is

646 a major determinant of mRNA stability. Cell. 2015;

647 32. Radhakrishnan A, Chen YH, Martin S, Alhusaini N, Green R, Coller J. The DEAD-Box

648 Protein Dhh1p Couples mRNA Decay and Translation by Monitoring Codon Optimality. Cell.

649 2016;

650 33. Duret L, Mouchiroud D. Expression pattern and, surprisingly, gene length shape codon usage 
651 in Caenorhabditis, Drosophila, and Arabidopsis. Proc Natl Acad Sci U S A. 1999;

652 34. Hiraoka Y, Kawamata K, Haraguchi T, Chikashige Y. Codon usage bias is correlated with

653 gene expression levels in the fission yeast Schizosaccharomyces pombe. Genes to Cells. 2009;

654 35. Sahoo S, Das SS, Rakshit R. Codon usage pattern and predicted gene expression in

655 Arabidopsis thaliana. Gene X. Elsevier; 2019;2:100012.

656 36. Payne BL, Alvarez-Ponce D. Codon usage differences among genes expressed in different

657 tissues of drosophila melanogaster. Genome Biol Evol. 2019;

658 37. Das S, Chottopadhyay B, Sahoo S. Comparative analysis of predicted gene expression

659 among crenarchaeal genomes. Genomics Inform. Korea Genome Organization; 2017;15:38.

660 38. Roymondal U, Das S, Sahoo S. Predicting gene expression level from relative codon usage

661 bias: An application to escherichia coli genome. DNA Res. 2009;

662 39. Roller M, Lucić V, Nagy I, Perica T, Vlahoviček K. Environmental shaping of codon usage

663 and functional adaptation across microbial communities. Nucleic Acids Res. 2013;

664 40. Angione C, Lió P. Predictive analytics of environmental adaptability in multi-omic network 665 models. Sci Rep. 2015;

666 41. Badet T, Peyraud R, Mbengue M, Navaud O, Derbyshire M, Oliver RP, et al. Codon 667 optimization underpins generalist parasitism in fungi. Elife. 2017;

668 42. Hart A, Cortés MP, Latorre M, Martinez S. Codon usage bias reveals genomic adaptations to 669 environmental conditions in an acidophilic consortium. PLoS One. 2018; 
43. Okie JG, Poret-Peterson AT, Lee ZMP, Richter A, Alcaraz LD, Eguiarte LE, et al. Genomic

671 adaptations in information processing underpin trophic strategy in a whole-ecosystem nutrient

672 enrichment experiment. Elife. 2020;

673 44. Sellick CA, Campbell RN, Reece RJ. Chapter 3 Galactose Metabolism in Yeast-Structure

674 and Regulation of the Leloir Pathway Enzymes and the Genes Encoding Them. Int. Rev. Cell

675 Mol. Biol. 2008.

676 45. CAPUTTO R, LELOIR LR. The enzymatic transformation of galactose into glucose

677 derivatives. J Biol Chem. 1949;

678 46. Hashimoto H, Kikuchi Y, Nogi Y, Fukasawa T. Regulation of expression of the galactose

679 gene cluster in Saccharomyces cerevisiae. Mol Gen Genet MGG. 1983;

681 genomics of biotechnologically important yeasts. Proc Natl Acad Sci U S A. 2016;

682 48. Slot JC, Rokas A. Multiple GAL pathway gene clusters evolved independently and by 683 different mechanisms in fungi. Proc Natl Acad Sci U S A. 2010;

684 49. Dalal CK, Zuleta IA, Mitchell KF, Andes DR, El-Samad H, Johnson AD. Transcriptional 685 rewiring over evolutionary timescales changes quantitative and qualitative properties of gene 686 expression. Elife. 2016;

687 50. Martchenko M, Levitin A, Hogues H, Nantel A, Whiteway M. Transcriptional Rewiring of 688 Fungal Galactose-Metabolism Circuitry. Curr Biol. 2007;

689 51. Kuang MC, Hutchins PD, Russell JD, Coon JJ, Hittinger CT. Ongoing resolution of duplicate 
690 gene functions shapes the diversification of a metabolic network. Elife. 2016;

691 52. Roop JI, Chang KC, Brem RB. Polygenic evolution of a sugar specialization trade-off in

692 yeast. Nature. 2016;

693 53. Hittinger CT, Rokas A, Carroll SB. Parallel inactivation of multiple GAL pathway genes and 694 ecological diversification in yeasts. Proc Natl Acad Sci U S A. 2004;

695 54. Opulente DA, Rollinson EJ, Bernick-Roehr C, Hulfachor AB, Rokas A, Kurtzman CP, et al.

696 Factors driving metabolic diversity in the budding yeast subphylum. BMC Biol. 2018;

697 55. Ferea TL, Botstein D, Brown PO, Rosenzweig RF. Systematic changes in gene expression 698 patterns following adaptive evolution in yeast. Proc Natl Acad Sci U S A. 1999;

699 56. Fraser HB, Moses AM, Schadt EE. Evidence for widespread adaptive evolution of gene 700 expression in budding yeast. Proc Natl Acad Sci U S A. 2010;

701 57. Thompson DA, Cubillos FA. Natural gene expression variation studies in yeast. Yeast. 2017;

702 58. Shen XX, Opulente DA, Kominek J, Zhou X, Steenwyk JL, Buh K V., et al. Tempo and

703 Mode of Genome Evolution in the Budding Yeast Subphylum. Cell. 2018;

704 59. Labella AL, Opulente DA, Steenwyk JL, Hittinger CT, Rokas A. Variation and selection on 705 codon usage bias across an entire subphylum. PLoS Genet. 2019;

706 60. Kurtzman, C.P., Fell JW. The yeasts a taxanomic study 5th edn. Elsevier Science Pulishers, 707 Amsterdam. The Yeasts. 2011.

708 61. Kanehisa M, Sato Y, Kawashima M, Furumichi M, Tanabe M. KEGG as a reference 
709 resource for gene and protein annotation. Nucleic Acids Res. 2016;

710 62. Wolfe KH, Shields DC. Molecular evidence for an ancient duplication of the entire yeast

711 genome. Nature. 1997;

712 63. Hittinger CT, Carroll SB. Gene duplication and the adaptive evolution of a classic genetic

713 switch. Nature. 2007;

714 64. Kalyaanamoorthy S, Minh BQ, Wong TKF, Von Haeseler A, Jermiin LS. ModelFinder: Fast

715 model selection for accurate phylogenetic estimates. Nat Methods. 2017;

716 65. Trifinopoulos J, Nguyen LT, von Haeseler A, Minh BQ. W-IQ-TREE: a fast online

717 phylogenetic tool for maximum likelihood analysis. Nucleic Acids Res. 2016;

718 66. Nguyen LT, Schmidt HA, Von Haeseler A, Minh BQ. IQ-TREE: A fast and effective

719 stochastic algorithm for estimating maximum-likelihood phylogenies. Mol Biol Evol. 2015;

720 67. Sabi R, Daniel RV, Tuller T. StAIcalc: TRNA adaptation index calculator based on species-

721 specific weights. Bioinformatics. 2017;

722 68. Bu L, Bergthorsson U, Katju V. Local synteny and codon usage contribute to asymmetric

723 sequence divergence of Saccharomyces cerevisiae gene duplicates. BMC Evol Biol. 2011;

724 69. Felsenstein J. Phylogenies and the comparative method. Am Nat. 1985;

725 70. Dray S, Dufour AB. The ade4 package: Implementing the duality diagram for ecologists. J

726 Stat Softw. 2007;

727 71. Blomberg SP, Lefevre JG, Wells JA, Waterhouse M. Independent contrasts and PGLS 
728 regression estimators are equivalent. Syst. Biol. 2012.

729 72. Garland T, Ives AR. Using the past to predict the present: Confidence intervals for regression

730 equations in phylogenetic comparative methods. Am Nat. 2000;

731 73. Spencer J, Rawling S, Stratford M, Steels H, Novodvorska M, Archer DB, et al. Yeast

732 identification: Reassessment of assimilation tests as sole universal identifiers. Lett Appl

733 Microbiol. 2011;

734 74. Pincus DH, Orenga S, Chatellier S. Yeast identification - Past, present, and future methods.

735 Med. Mycol. 2007.

736 75. Lopandic K, Zelger S, Bánszky LK, Eliskases-Lechner F, Prillinger H. Identification of

737 yeasts associated with milk products using traditional and molecular techniques. Food Microbiol.

738 2006;

739 76. Dewan S, Tamang JP. Microbial and analytical characterization of Chhu - A traditional

740 fermented milk product of the Sikkim Himalayas. J Sci Ind Res (India). 2006;

741 77. WILCOXON F. Individual comparisons of grouped data by ranking methods. J Econ

742 Entomol. 1946;

743 78. Yang Z. Paml: A program package for phylogenetic analysis by maximum likelihood.

744 Bioinformatics. 1997;

745 79. Yang Z. PAML 4: Phylogenetic analysis by maximum likelihood. Mol Biol Evol. 2007;

746 80. Abascal F, Zardoya R, Telford MJ. TranslatorX: Multiple alignment of nucleotide sequences

747 guided by amino acid translations. Nucleic Acids Res. 2010; 
81. Kuang MC, Kominek J, Alexander WG, Cheng JF, Wrobel RL, Hittinger CT. Repeated cis-

749 regulatory tuning of a metabolic bottleneck gene during evolution. Mol Biol Evol. 2018;

750 82. Hittinger CT, Gonçalves P, Sampaio JP, Dover J, Johnston M, Rokas A. Remarkably ancient

751 balanced polymorphisms in a multi-locus gene network. Nature. 2010;

752 83. Bustamante CD, Nielsen R, Hartl DL. A maximum likelihood method for analyzing

753 pseudogene evolution: Implications for silent site evolution in humans and rodents. Mol Biol

754 Evol. 2002;

755 84. Miyata K, Hayashida H. Extraordinarily high evolutionary rate of pseudogenes: Evidence for

756 the presence of selective pressure against changes between synonymous codons. Proc Natl Acad

757 Sci U S A. 1981;

758 85. Singh V, Satheesh S V., Raghavendra ML, Sadhale PP. The key enzyme in galactose

759 metabolism, UDP-galactose-4-epimerase, affects cell-wall integrity and morphology in Candida

760 albicans even in the absence of galactose. Fungal Genet Biol. 2007;

761 86. Matsuzawa T, Fujita Y, Tanaka N, Tohda H, Itadani A, Takegawa K. New insights into

762 galactose metabolism by Schizosaccharomyces pombe: Isolation and characterization of a

763 galactose-assimilating mutant. J Biosci Bioeng. 2011;

764 87. Bulmer M. The selection-mutation-drift theory of synonymous codon usage. Genetics. 1991;

765 88. The rate of synonymous substitution in enterobacterial genes is inversely related to codon

766 usage bias. Mol Biol Evol. 1987; 
768 analysis reveals the correlation between codon usage bias and gene expression in Cuscuta

769 australis. Genomics. Elsevier; 2020;

770 90. Astro V, Asperti C, Cangi MG, Doglioni C, de Curtis I. Liprin-alpha1 regulates breast cancer

771 cell invasion by affecting cell motility, invadopodia and extracellular matrix degradation.

772 Oncogene [Internet]. 2010/12/15. 2011;30:1841-9. Available from:

773 https://www.ncbi.nlm.nih.gov/pubmed/21151172

774 91. Zhoua Z, Danga Y, Zhou M, Li L, Yu CH, Fu J, et al. Codon usage is an important

775 determinant of gene expression levels largely through its effects on transcription. Proc Natl Acad

776 Sci U S A. 2016;

777 92. DOUGLAS HC, HAWTHORNE DC. ENZYMATIC EXPRESSION AND GENETIC

778 LINKAGE OF GENES CONTROLLING GALACTOSE UTILIZATION IN

779 SACCHAROMYCES. Genetics. 1964;

780 93. De Jongh WA, Bro C, Ostergaard S, Regenberg B, Olsson L, Nielsen J. The roles of

781 galactitol, galactose-1-phosphate, and phosphoglucomutase in galactose-lnduced toxicity in

782 Saccharomyces cerevisiae. Biotechnol Bioeng. 2008;

783 94. Opulente DA, Langdon QK, Buh K V., Haase MAB, Sylvester K, Moriarty R V., et al.

784 Pathogenic budding yeasts isolated outside of clinical settings. FEMS Yeast Res. 2019;

785 95. Gutiérrez J, Morales P, González MA, Quindós G. Candida dubliniensis, a new fungal

786 pathogen. J Basic Microbiol. 2002;

787 96. Lockhart SR, Messer SA, Pfaller MA, Diekema DJ. Identification and susceptibility profile 
788 of Candida fermentati from a worldwide collection of Candida guilliermondii clinical isolates. J

789 Clin Microbiol. 2009;

790 97. Wingard JR, Merz WG, Saral R. Candida tropicalis: A major pathogen in

791 immunocompromised patients. Ann Intern Med. 1979;

792 98. Papon N, Courdavault V, Clastre M, Bennett RJ. Emerging and Emerged Pathogenic

793 Candida Species: Beyond the Candida albicans Paradigm. PLoS Pathog. 2013;

794 99. Gargeya IB, Pruitt WR, Simmons RB, Meyer SA, Ahearn DG. Occurrence of Clavispora

795 lusitaniae, the teleomorph of Candida lusitaniae, among clinical isolates. J Clin Microbiol. 1990;

796 100. Rosenbach A, Dignard D, Pierce J V., Whiteway M, Kumamoto CA. Adaptations of

797 Candida albicans for growth in the mammalian intestinal tract. Eukaryot Cell. 2010;

798 101. Cadete RM, Santos RO, Melo MA, Mouro A, Gonçalves DL, Stambuk BU, et al.

799 Spathaspora arborariae sp. nov., a d-xylose-fermenting yeast species isolated from rotting wood

800 in Brazil. FEMS Yeast Res. 2009;

801 102. Lopes MR, Morais CG, Kominek J, Cadete RM, Soares MA, Uetanabaro APT, et al.

802 Genomic analysis and D-xylose fermentation of three novel Spathaspora species: Spathaspora

803 girioi sp. nov., Spathaspora hagerdaliae f. a., sp. nov. and spathaspora gorwiae f. a., sp. nov.

804 FEMS Yeast Res. 2016;

805 103. Tekaia F, Latgé JP. Aspergillus fumigatus: Saprophyte or pathogen? Curr. Opin. Microbiol. 8062005.

807 104. May RC, Stone NRH, Wiesner DL, Bicanic T, Nielsen K. Cryptococcus: From 
808 environmental saprophyte to global pathogen. Nat. Rev. Microbiol. 2016.

809 105. Giraffa G, Chanishvili N, Widyastuti Y. Importance of lactobacilli in food and feed

810 biotechnology. Res Microbiol. 2010;

811 106. Hittinger CT, Steele JL, Ryder DS. Diverse yeasts for diverse fermented beverages and 812 foods. Curr. Opin. Biotechnol. 2018.

813 107. Andrés López-Arboleda W, Ramírez-Castrillón M, Mambuscay-Mena LA, Osorio-Cadavid

814 E. Diversidad de levaduras asociadas a chichas tradicionales de Colombia Yeast diversity

815 associated to Colombian traditional " chichas ". Rev Colomb Biotecnol Diciembre. 2010;

816 108. Coton E, Coton M, Levert D, Casaregola S, Sohier D. Yeast ecology in French cider and

817 black olive natural fermentations. Int J Food Microbiol. 2006;

818 109. Marsh AJ, O’Sullivan O, Hill C, Ross RP, Cotter PD. Sequence-based analysis of the

819 bacterial and fungal compositions of multiple kombucha (tea fungus) samples. Food Microbiol. $820 \quad 2014$

821 110. Kirchmayr MR, Segura-García LE, Lappe-Oliveras P, Moreno-Terrazas R, de la Rosa M,

822 Gschaedler Mathis A. Impact of environmental conditions and process modifications on

823 microbial diversity, fermentation efficiency and chemical profile during the fermentation of

824 Mezcal in Oaxaca. LWT - Food Sci Technol. 2017;

825 111. Maïworé J, Tatsadjieu Ngoune L, Piro-Metayer I, Montet D. Identification of yeasts present

826 in artisanal yoghurt and traditionally fermented milks consumed in the northern part of

827 Cameroon. Sci African. 2019; 
828 112. Garnier L, Valence F, Pawtowski A, Auhustsinava-Galerne L, Frotté N, Baroncelli R, et al.

829 Diversity of spoilage fungi associated with various French dairy products. Int J Food Microbiol.

$830 \quad 2017$

831 113. Koutinas AA, Papapostolou H, Dimitrellou D, Kopsahelis N, Katechaki E, Bekatorou A, et

832 al. Whey valorisation: A complete and novel technology development for dairy industry starter

833 culture production. Bioresour Technol. 2009;

834 114. Hershberg R, Petrov DA. General rules for optimal codon choice. PLoS Genet. 2009;

835 115. Opulente DA, Morales CM, Carey LB, Rest JS. Coevolution Trumps Pleiotropy: Carbon

836 Assimilation Traits Are Independent of Metabolic Network Structure in Budding Yeast. PLoS

837 One. 2013;

838 116. Carr FJ, Chill D, Maida N. The lactic acid bacteria: A literature survey. Crit. Rev.

839 Microbiol. 2002.

840 117. Hohmann S, Meacock PA. Thiamin metabolism and thiamin diphosphate-dependent

841 enzymes in the yeast Saccharomyces cerevisiae: genetic regulation. Biochim. Biophys. Acta -

842 Protein Struct. Mol. Enzymol. 1998.

843 118. Viljoen BC. The interaction between yeasts and bacteria in dairy environments. Int J Food

844 Microbiol. 2001.

845 119. Rinke C, Schwientek P, Sczyrba A, Ivanova NN, Anderson IJ, Cheng JF, et al. Insights into

846 the phylogeny and coding potential of microbial dark matter. Nature. 2013; 